\title{
Excellent UV-Absorbing Cotton Fabric with High Durability and Hydrophobicity Based on Surface- Initiated ATRP of Polymerizable Organic UV- Absorber
}

\author{
Saixiang Hua \\ Anhui University \\ Huichao Zhu \\ Anhui University \\ Xiao Wang \\ Anhui University \\ Mingyuan Wu \\ Anhui University \\ Qingyun Wu \\ Anhui University \\ Jiuyi Liu \\ Anhui University \\ Jianjun Yang \\ Anhui University \\ Jianan Zhang ( $\boldsymbol{\nabla}$ jianan@ahu.edu.cn ) \\ Anhui University https://orcid.org/0000-0003-3195-9882
}

\section{Research Article}

Keywords: UV absorption performance, durability, SI-ATRP, UV protection factor, hydrophobicity

Posted Date: August 13th, 2021

DOI: https://doi.org/10.21203/rs.3.rs-729544/v1

License: (c) (i) This work is licensed under a Creative Commons Attribution 4.0 International License. Read Full License 


\section{Abstract}

Ultraviolet (UV) protective cotton fabric is a convenient and reliable way to protect human body from sunlight. Herein, a polymerizable organic UV-absorber, 2-hydroxy-4-(3-methacryloxy-2-hydroxylpropoxy) benzophenone (BPMA) was prepared from the ring-opening addition reaction of 2,4dihydroxybenzophenone (UV-0) with glycidyl methacrylate (GMA). The initiators tethered cotton fabric (CF-Br) were prepared by the immobilization of a-bromoisobutyryl groups. PBPMA grafted cotton fabric (CF-g-PBPMA) was fabricated via surface-initiated atom transfer radical polymerization (SI-ATRP). The experimental results indicated that BPMA was successfully synthesized, and PBPMA chains were successfully grafted from CF-Br. Importantly, CF-g-PBPMA exhibited excellent UV absorption performance, hydrophobic ability and outstanding durability. Compared with the UV protection factor (UPF) of the original cotton fabric (3.5), the UPF of CF-g-PBPMA achieved 241.5, which was far beyond the excellent protection UPF rating (50+) and was up to 69-fold higher than that of original cotton fabric. Compared with the water contact angle (WCA) of the original cotton fabric of $46^{\circ}$, the WCA of CF-g-PBPMA reached $140^{\circ}$, showing excellent hydrophobic property. In addition, after five washing cycles, CF-g-PBPMA showed an outstanding durability with the WCA and UPF achieving $131^{\circ}$ and 226.9 , respectively.

\section{Introduction}

Nowadays, the level of ultraviolet (UV) radiation reaching the Earth's surface has increased due to the ozone depletion and other environmental related issues. UV protective cotton fabric is a convenient and reliable way to protect human body from sunlight (Gorjanc et al. 2014). The protection performance of cotton fabric against UV radiation can be quantified by measuring the UV protection factor (UPF) in vitro (Chen and Yin 2010; Oda 2011). According to the Australian/New Zealand Standard AS/NZS 4399:1996, the UPF values of $15-24,25-39$, and above 40 (where higher than 50 is called $50+$ ) mean good, very good, and excellent UV protection performance for cotton fabric, respectively (Algaba and Riva 2002).

White or light color dyed cotton fabric is very popular with consumers in summer. The UPF value of such cotton fabric is very poor (Czajkowski et al. 2006). UV light absorbers (UVA), which can convert the absorbed radiation energy into less harmful thermal energy through a photophysical process involving ground state and excited state molecules (Entezaria et al. 2012; Waiblinger et al. 1999), are often applied to enhance the UV absorption performance of cotton fabric by pad-dry-cure (Abd et al. 2013; Ahmad et al. 2019; Rong et al. 2015), hydrothermal (Ates and Unalan 2012), graft (Shen et al. 2014), sol-gel (Gupta et al. 2021), dip coating (Gouda and Keshk 2010; Ren et al. 2018; Wang et al. 2011) and self-assembly (Abd et al. 2020; Cheng et al. 2018) methods, etc. UVA include benzophenone (Oda 2011; Shen et al. 2016), benzotriazole (Oda 2011; Suhadolnik et al. 2002), cyanoacrylate (Choi and Song 2008), hindered amine (Bojinov 2006), triazine related compounds (Czajkowski et al. 2012) and metal oxide nanomaterials (Ibrahim et al. 2013) etc. Benzotriazole and benzophenone are the most commonly used among the above UVA (Shen et al. 2014). However, these UVA have no reactive group, and lack affinity for cellulose fibers. When applied to cotton fabric, they have poor wash fastness (Stark and Matuana 2006; Shen et al. 2014). In order to improve the durability of cotton fabric, UVA of the "reactive type" containing reactive 
groups formed covalent bonds with cellulose after fixation on this fiber. Although the modified cotton fabric maintained durable UV protective performance, the UPF values still need further enhancement (Akrman and Prikrryl 2008; Czajkowski 2012; Ibrahim et al. 2020; Mamnicka and Czajkowski 2012; Shen et al. 2014).

In this paper, we put forward a novel and efficient method for preparing UV-absorbing cotton fabric with high durability and hydrophobicity. A polymerizable organic UV-absorber, 2-hydroxy-4-(3-methacryloxy-2hydroxylpropoxy) benzophenone (BPMA) has been prepared from the ring-opening addition reaction of 2,4-dihydroxybenzophenone (UV-0) with glycidyl methacrylate (GMA) (Dong et al. 2018;2018a;2018b; Zhao and Dan 2007). The initiators tethered cotton fabric (CF-Br) was prepared by the immobilization of a-bromoisobutyryl groups. PBPMA grafted cotton fabric (CF-g-PBPMA) was fabricated via surfaceinitiated atom transfer radical polymerization (SI-ATRP). The UV absorption performance, surface hydrophobicity, and durability of CF-g-PBPMA were investigated.

\section{Experimental Section}

\section{Materials}

Cotton fabric (coarse sheeting, cellulose content $=98 \%$, porosity $=16 \%$ ) without further bleaching was purchased from Manheng Textile Ltd. Co. (Hefei, China). 2,4-Dihydroxybenzophenone (UV-0, 99\%) and glycidyl methacrylate (GMA, 97\%) were bought from Sinopharm Chemical Reagent, Ltd. Co. aBromoisobutyryl bromide (BiBB, 99\%), 2-bromoisobutyric acid, 2,2'-bipyridyl (bpy, 99\%), ascorbic acid (AscA, $\geq 99 \%$ ), N,N-dimethylformamide (DMF), cyclohexane, alcohol ethoxylate (AEO), triethylamine and copper (II) bromide $\left(\mathrm{CuBr}_{2}, 99.9 \%\right)$ were obtained from Sinopharm Chemical Reagent Company Ltd.. Sodium hydroxide ( $\mathrm{NaOH}, 99 \%)$, methanol, ethanol, ethyl acetate (EA, 99.5\%), sulfuric acid (98\%) and petroleum ether (PE, 99.5\%) were purchased from Sigma-Aldrich. The above chemical reagents were used without further purification. Deionized water was made in-house.

\section{Synthesis of 2-hydroxy-4-(3-methacryloxy-2-hydroxylpropoxy) benzophenone (BPMA)}

The procedure for preparing polymer grafted cotton fabric for UV-absorption was illustrated in Scheme 1.

2-Hydroxy-4-(3-methacryloxy-2-hydroxylpropoxy) benzophenone (BPMA) was synthesized (Scheme 1) as described in an earlier literature (Zhao and Dan 2007). A mixture of $\mathrm{NaOH}(0.155 \mathrm{~g}, 3.88 \mathrm{mmol}$ ) and UV-0 $(10.7 \mathrm{~g}, 0.05 \mathrm{~mol})$ were added into a $250 \mathrm{~mL}$ three-necked round bottom flask with a ventilation pipe, a stirring paddle and a refluxing condensation pipe. To exclude the oxygen gas, the reactor was deaired by vacuum pump and was aerated with nitrogen gas, and then, GMA $(7.81 \mathrm{~g}, 0.055 \mathrm{~mol})$ was introduced into the reactor by syringe. The reaction was carried out at $80^{\circ} \mathrm{C}$ for about $5 \mathrm{~h}$. After the reaction was completed, a coarse product of yellow viscous liquid was obtained, which was dissolved in EA and washed with $1 \mathrm{wt} \%$ dilute sulfuric acid. The EA was removed under reduced pressure. Then, the pure BPMA was collected by chromatography on silica gel using the mixed solvent of PE and EA $(8: 1, v / v)$ as 
eluent. After complete removal of solvents under vacuum, $8.45 \mathrm{~g}$ of BPMA were obtained with the yield of $74 \%$.

\section{Preparation of initiator immobilized cotton fabric by esterification}

The original cotton fabric was thoroughly washed with deionized water, ethanol and acetone in turn to remove dust, and then dried in an oven at $50{ }^{\circ} \mathrm{C}$ for 8 hours. Next, $2 \mathrm{~g}$ of dried cotton fabric were added into a $150 \mathrm{ml}$ three-necked round bottom flask containing triethylamine $(2 \mathrm{ml})$ and DMF $(50 \mathrm{ml})$, and then $2 \mathrm{ml}$ of BIBB were added dropwise under mild magnetic stirring. After the reaction was carried out for 48 $\mathrm{h}$ at $20^{\circ} \mathrm{C}$, the modified cotton fabric was rinsed thoroughly by ethanol until the washed liquid became clear color. The initiator immobilized cotton fabric was obtained by drying in vacuum oven at $40^{\circ} \mathrm{C}$ for 16 $\mathrm{h}$ and was defined as CF-Br.

\section{Fabrication of PBPMA grafted cotton fabric by SI-ATRP}

$2 \mathrm{~g}$ of CF-Br was added to Schlenk flask containing methanol $(40 \mathrm{~mL})$ and 2-bromoisobutyric acid (0.012 $\mathrm{g}$, as the sacrificial initiator) solution. Under gentle magnetic stirring, BPMA (5 g), $\mathrm{CuBr}_{2}(2.6 \mathrm{mg})$, and bpy (7.2 mg) were sequentially added to Schlenk flask. After bubbling with $\mathrm{N}_{2}$ for $0.5 \mathrm{~h}$ under mild magnetic stirring, AscA ( $4.8 \mathrm{mg}$ ) was added to the reactor and the polymerization was carried out at $60{ }^{\circ} \mathrm{C}$ for $12 \mathrm{~h}$. The obtained cotton fabric was collected and washed with methanol to remove the free PBPMA and BPMA. The PBPMA grafted cotton fabric was then dried in an oven at $40^{\circ} \mathrm{C}$ for $24 \mathrm{~h}$, which was defined as CF-g-PBPMA. The free PBPMA homopolymer was obtained by precipitation into cyclohexane for GPC characterization. The grafting ratio (G\%) of CF-g-PBPMA was calculated according to the following formula to be $18 \%$.

$$
G \%=\frac{W_{g}-W_{0}}{W_{0}} \times 100 \%
$$

where $\mathrm{W}_{\mathrm{g}}$ and $\mathrm{W}_{0}$ are weights of CF-g-PBPMA and CF-Br, respectively.

\section{Characterization}

Using dimethyl sulfoxide-d6 as solvent, high resolution proton nuclear magnetic resonance (1H-NMR) spectrum was recorded on a Varian INO-VA-400 spectrometer of $400 \mathrm{MHz}$. The surface compositions of cotton fabric samples were characterized by attenuated total reflectance Fourier transform infrared spectroscopy (ATR-FTIR) on a Perkin-Elmer Spectrum 2000 Fourier transform infrared spectrometer (Specac Ltd). The surface morphologies of modified membranes were by a field-emitting scanning electron microscope (FESEM, XL 30S-FEG, Holland) at an acceleration voltage of $10 \mathrm{kV}$. All the samples were coated with gold. The molecular weight and molecular weight distribution ( $Đ)$ of the free PBPMA homopolymer were determined by gel permeation chromatograph (GPC; HLC-8320), and the measurements were conducted with differential refractometer using columns (TSK gel super HZM-M and TSK gel SuperHZ3000 in series) with THF as the eluent $\left(35^{\circ} \mathrm{C}\right.$, flow rate of $\left.1 \mathrm{~mL} / \mathrm{min}\right)$ with diphenyl ether 
used as internal references. Linear polystyrene standard was used for calibration. The water contact angle (WCA) tests were carried out with contact angle measuring instrument (DSAIOMKZ, China), and each sample was tested at more than three different locations. UV-visible absorption spectra of samples were measured were measured by using a UV-visible UH5300 spectrophotometer (Hitachi, Tokyo, Japan). According to the Australian/New Zealand Standard AS/NZS 4399:1996, each specimen of the treated cotton fabric samples was cut at least $5 \times 5 \mathrm{~cm}$ and placed against the sample transmission port opening in the sphere, and were examined for their UV absorption performance by Cary 5000 UV-visible Spectrophotometer (Agilent, America). The UPF was calculated by using mean percentage transmission in the UVB region $(280-315 \mathrm{~nm})$ and UVA region $(315-400 \mathrm{~nm})$ according to the following formula:

$$
U P F=\frac{\sum_{280 n m}^{400 n m} E(\lambda) S(\lambda) \Delta \lambda}{\sum_{280 n m}^{400 n m} E(\lambda) S(\lambda) T(\lambda) \Delta \lambda}
$$

In this formula, $E(\lambda)=$ relative erythemal spectral effectiveness, $S(\lambda)=$ solar spectral irradiance in $\left[\mathrm{W} . \mathrm{cm}^{-2}\right.$ $\left.\mathrm{nm}^{-1}\right], \mathrm{T}(\lambda)$ is spectral transmittance of the item, $\lambda$ is wavelength step [nm], $\Delta \lambda$ is the interval of wavelength $[\mathrm{nm}]$.

Laundering durability was performed to evaluate the stability of surface hydrophobicity and UV absorption performance of the cotton fabric samples in analogy to the ISO 105-C02:1989(E) method. Samples of cotton fabric were immersed into an aqueous solution containing $5 \mathrm{~g} / \mathrm{L}$ AEO nonionic detergent. The bath was thermostatically adjusted to $50{ }^{\circ} \mathrm{C}$. The test was run for $45 \mathrm{~min}$ at $750 \mathrm{rpm}$. The samples of cotton fabric were then removed, rinsed in cold distilled water for 10 minutes and finally dried at room temperature for $12 \mathrm{~h}$. Samples of cotton fabric were washed for one and five cycles.

\section{Results And Discussion}

\section{Fabrication of CF-g-PBPMA}

Because the surface of the original cotton fabric contains a lot of active hydroxyl groups, it is possible to modify the original cotton fabric (Scheme 2a). After treatment with BiBB, a-bromoisobutyryl groups bonded cotton fabric (CF-Br) was obtained with initiating sites for ATRP (Scheme $2 b$ ). Then, PBPMA was grafted from the surfaces of CF-Br fibers to obtain the robust and durable CF-g-PBPMA for efficient UV absorption (Scheme 2c)

\section{Structure and performance characterization of BPMA}

In order to further determine the chemical structure of BPMA, BPMA was characterized by $1 \mathrm{H}-\mathrm{NMR}$. As shown in Fig. 1a, the hydrogen atoms of BPMA were marked using 1, 2, 3, 4, 5, 6, 7, 8, 9, 10, d, e, f, g, h, k. The peaks at $\delta=7.29-7.60 \mathrm{ppm}$ were vested to aromatic protons $\left(\mathrm{H}_{1}, \mathrm{H}_{2}, \mathrm{H}_{3}, \mathrm{H}_{4}, \mathrm{H}_{5}\right.$ and $\left.\mathrm{H}_{6}\right)$ and the peaks at $\delta=6.36-6.55 \mathrm{ppm}$ were also belonged to aromatic protons $\left(\mathrm{H}_{7}\right.$ and $\left.\mathrm{H}_{8}\right)$. The weak peak at $5.42 \mathrm{ppm}$ was due to $\mathrm{H}_{9}$. The peak at $11.95 \mathrm{ppm}$ was responsible for the proton of phenolic hydroxyl $\left(\mathrm{H}_{10}\right)$. The 
signals at $\delta=5.66$ and $6.05 \mathrm{ppm}$ were assigned to $\mathrm{H}_{\mathrm{e}}$ and $\mathrm{H}_{\mathrm{f}}$, respectively. The peaks at $\delta=4.11-4.30 \mathrm{ppm}$ pertained to five protons of $-\mathrm{CH}_{2}-\mathrm{CH}-\mathrm{CH}_{2}-\left(\mathrm{H}_{\mathrm{g}}, \mathrm{H}_{\mathrm{h}}\right.$ and $\left.\mathrm{H}_{\mathrm{k}}\right)$ and peaks at about $\delta=1.86 \mathrm{ppm}$ belonged to three protons of $-\mathrm{CH}_{3}\left(\mathrm{H}_{\mathrm{d}}\right)$.

To further confirm ring-opening addition reaction of UV-0 with GMA, the chemical information of UV-0 and BPMA was analyzed by FT-IR (Fig. 2). Compared with UV-0, new peaks appeared in the spectrum of BPMA at 1724 and $1024 \mathrm{~cm}^{-1}$ corresponding to the $\mathrm{C}=\mathrm{O}$ stretching vibration and $-\mathrm{CHOH}$, respectively, which indicated the successful synthesis of BPMA.

The UV absorption performance of BPMA and UV-0 was evaluated by UV absorption spectra. As shown in Fig. 3, the peak at $240 \mathrm{~nm}$ in the UV absorption spectrum of BPMA was the $\pi \rightarrow \pi^{\star}$ transition which was similar to UV-0. In the UV spectrum of BPMA, a hypsochomic shift from $292 \mathrm{~nm}$ to $287 \mathrm{~nm}$ was the $\pi \rightarrow \pi^{*}$ transition due to the newly appearance of an ether bond Ar-O-C, which was formed by the ringopening addition reaction between 4-position hydroxyl of UV-0 and GMA. The UV absorption band of BPMA at $322 \mathrm{~nm}$ corresponded to the $\mathrm{n} \rightarrow \pi^{\star}$ transition of carbonyl, which was also similar to that of UV0 . The three peaks at 240,287 , and $322 \mathrm{~nm}$ in the UV absorption spectrum of BPMA confirmed the successful ring-opening addition reaction between UV-0 and GMA, which would retain the excellent UV absorption performance of UV-0.

\section{Microstructure, surface hydrophobicity and chemical compositions of CF-g-PBPMA}

To further confirm the initiator tethering and subsequent PBPMA grafting, the pristine cotton fabric, $\mathrm{CF}-\mathrm{Br}$, and CF-g-PBPMA were characterized by FT-IR to identify the chemical variation of cotton fabric during the whole synthetic process, respectively, (Fig. 4). Compared with the spectrum of original cotton fabric (Fig. 4a), a new peak at $1717 \mathrm{~cm}^{-1}$ corresponding to the $C=0$ stretching vibration verified that the active hydroxyl groups on the surface of the original fabric were successfully esterified with BIBB. Therefore, the introduced a-bromoisobutyryl groups on the surface of cotton fabric were acted as ATRP initiating sites (Fig. 4b). As shown in Fig. 4c, a new absorption band located at $1254 \mathrm{~cm}^{-1}$ in the spectrum of CF-gPBPMA pertained to the C-O-C after the polymer was grafted. The significantly enhanced peaks at 1724 and $1625 \mathrm{~cm}^{-1}$ corresponded ester carbonyl and ketone carbonyl. In addition, the peaks located at 1502 and $704 \mathrm{~cm}^{-1}$ were attributed to the aromatic skeletal vibration of benzene ring and $\mathrm{C}-\mathrm{H}$ out-of-plane bending vibration of monosubstituted benzene rings, respectively. By using 2-bromoisobutyric acid as sacrificial initiator, the $M_{n}$ and $€$ of grafted PBPMA were determined to be 9214 and 1.3, respectively, which confirmed the controlled polymer grafting.

The surface structures of original cotton fabric and modified cotton fabric were illustrated via SEM analyses (Fig. 5). The original cotton fabric presented a typically smooth surface (Fig. 5a and b). After being treated with $\mathrm{BiBB}$, there was no significant difference between the surface smoothness of CF-Br (Fig. $5 \mathrm{c}$ and d) and that of original cotton fabric. Compared with relatively smooth surface of CF-Br, the irregular and rough folds were observed on the surface of CF-g-PBPMA (Fig. 5e and f). The highly 
irregular wrinkles and a greater surface roughness were originated from the accumulation of grafted PBPMA chains and thus confirmed the successful polymer grafting.

In order to evaluate the surface hydrophobicity of cotton fabric, the water contact angles (WCAs) were examined and illustrated in Fig. 6 . When the water droplet approached the original cotton fabric, it could be observed that the WCA was about $46^{\circ}$ (Fig. 6a). However, the water droplet was adsorbed completely within $10 \mathrm{~s}$ owing to original cotton fabric was composed of cellulose fibers with massive surface hydroxyl groups. After initiator tethering, the immobilized a-bromoisobutyryl groups enabled the hydrophilic cotton fibers to exhibit hydrophobicity with a WCA of about $121^{\circ}$ (Fig. 6b). After PBPMA grafting, the WCA of CF-g-PBPMA increased from 121 to $140^{\circ}$ due to the increased surface roughness of cotton fabric induced by the accumulated PBPMA (Fig. 6c).

\section{UV absorption performance of CF-g-PBPMA}

The UV absorption performance of the original cotton fabric and CF-g-PBPMA was evaluated via the UPF value which was calculated by using mean percentage transmission in the UVB region $(280-315 \mathrm{~nm})$ and UVA region (315-400nm). Fig. 7 displayed the UV transmittance of the original cotton fabric and CF-gPBPMA between $280-400 \mathrm{~nm}$. The UPF value of original cotton fabric was only 3.5 , displaying not reaching enough UV protection performance between 280-400 nm. After PBPMA grafting, the UV transmission of CF-g-PBPMA decreased obviously and the UPF increased to 241.5, which was far beyond the excellent protection UPF rating (50+) and was up to 69-fold higher than original cotton fabric. The experimental results indicated that CF-g-PBPMA demonstrated excellent UV absorption performance ranging from $280-400 \mathrm{~nm}$.

\section{Durability analysis}

In order to evaluate the durability of CF-g-PBPMA, the UV absorption and surface hydrophobicity of CF-gPBPMA were tested after one/five cycles of washing. The WCAs of CF-g-P BPMA, after one/five cycles of washing, were shown in Fig.8. After one-cycle washed and five-cycles washed, the surface hydrophobicity of CF-g-PBPMA declined gradually. However, even after five washing cycles, the WCA of CF-g-PBPMA could still reach $131^{\circ}$.

Fig. 9 and Fig. 10 displayed the UV absorption performance of CF-g-PBPMA after one/five cycles of washing. Compared with CF-g-PBPMA, after one/five washing cycles, the UV-visible transmittance marginally increased in the range of $280-400 \mathrm{~nm}$ (Fig. 9). The reason can be obtained by analyzing Fig.10. Because the UV-visible absorption of CF-g-PBPMA between 280-400 nm decreased slightly after one/five cycles of washing. In addition, as can be seen from Fig. 9, even after five washing cycles, the UPF value of CF-g-PBPMA still achieved 226.9, which further demonstrated excellent durability of UV absorption performance.

Table 1 illustrated the UPF values of CF-g-PBPMA and other reported cotton fabric with UV absorption performance. Among all the UV protection fabrics, the UV absorption performance of CF-g-PBPMA 
possessed the highest UPF value (241.5). CF-g-PBPMA exhibited excellent performance of UV absorption with outstanding durability and hydrophobicity, which provides a novel and efficient strategy for the preparation of multifunctional fabrics.

Table 1 UPF values of various UV protection fabric.

\begin{tabular}{|c|c|c|c|c|}
\hline $\begin{array}{l}\text { Fabric } \\
\text { substrate }\end{array}$ & UVA & Treatment method & UPF & Ref. \\
\hline cotton & $\begin{array}{l}\text { benzophenone } \\
\text { (BPMA) }\end{array}$ & SI-ATRP (graft) & 241.5 & This study \\
\hline cotton & $\begin{array}{l}\text { metal oxide } \\
\text { nanomaterials }\end{array}$ & pad-dry-cure & 60 & (Abd et al. 2013) \\
\hline cotton & $\begin{array}{l}\text { metal oxide } \\
\text { nanomaterials }\end{array}$ & dip-pad-dry-cure & 155.7 & (Ahmad et al. 2019) \\
\hline cotton & benzophenone & pad-dry-cure & 36 & (Rong et al. 2015) \\
\hline cotton & benzophenone & graft & 52.1 & (Shen et al. 2014) \\
\hline cotton & $\begin{array}{l}\text { metal oxide } \\
\text { nanomaterials }\end{array}$ & coating & $>50$ & $\begin{array}{l}\text { (Gouda and Keshk } \\
\text { 2010) }\end{array}$ \\
\hline cotton & $\begin{array}{l}\text { metal oxide } \\
\text { nanomaterials }\end{array}$ & coating & 101.5 & (Wang et al 2011) \\
\hline cotton & $\begin{array}{l}\text { metal oxide } \\
\text { nanomaterials }\end{array}$ & layer-by-layer self-assembly & $>50$ & (Cheng et al. 2018) \\
\hline cotton & $\begin{array}{l}\text { metal oxide } \\
\text { nanomaterials }\end{array}$ & $\begin{array}{l}\text { electrostatic self-assembly } \\
\text { layer by layer }\end{array}$ & 189.2 & (Abd et al. 2020) \\
\hline cotton & benzotriazole & $\begin{array}{l}\text { dyeing with reactive dyes from } \\
\text { aqueous bath }\end{array}$ & 179 & $\begin{array}{l}\text { (Akrman and Přikryl } \\
\text { 2008) }\end{array}$ \\
\hline cotton & $\begin{array}{l}\text { triazine related } \\
\text { compounds }\end{array}$ & $\begin{array}{l}\text { exhaustion and dyeing from } \\
\text { aqueous bath }\end{array}$ & 118 & $\begin{array}{l}\text { (Mamnicka and } \\
\text { Czajkowski 2012) }\end{array}$ \\
\hline cotton & $\begin{array}{l}\text { triazine related } \\
\text { compounds }\end{array}$ & pad-dry & 58.2 & (Ibrahim et al.2020) \\
\hline
\end{tabular}

\section{Conclusions}

In this study, CF-g-PBPMA was successfully prepared by SI-ATRP method with synthetic BPMA as functional monomer. Compared with the UPF of the original cotton fabric (3.5), the UPF of CF-g-PBPMA achieved 241.5 which was far beyond the excellent protection UPF rating (50+) and was up to 69-fold higher than that of original cotton fabric. Compared with WCA of the original cotton fabric of $46^{\circ}, \mathrm{CF}-\mathrm{g}-$ PBPMA displayed excellent hydrophobic property with the WCA reaching $140^{\circ}$. The chemically grafted 
PBPMA enabled CF-g-PBPMA an outstanding durability with the WCA and UPF achieving $131^{\circ}$ and 226.9 after five washing cycles, respectively. The multifunctional CF-g-PBPMA has potential applications in various areas, such as medical, military, biological, and optoelectronic industrial fields.

\section{Declarations}

\section{Declaration of Competing Interest}

The authors declare no competing financial interest.

\section{Acknowledgment}

This work was financially supported by National Natural Science Foundation of China (51973001), the Major Program of University Natural Science Research Project of Anhui Province (KJ2019ZD01), the Key Projects of Innovation Program for Returned Scholars of Anhui Province (2019LCX006), and the Open fund for Discipline Construction, Institute of Physical Science and Information Technology, Anhui University.

\section{References}

1. Abd El-Hady MM et al. (2013). Flame retardancy and UV protection of cotton based fabrics using nano ZnO and polycarboxylic acids. Carbohydrate polymers 921:400-406.

2. Abd El-Hady MM et al. (2020). Highly hydrophobic and UV protective properties of cotton fabric using layer by layer self-assembly technique. Cellulose 272:1099-1110.

3. Ahmad I et at. (2019). Reactive Blue-25 dye $/ \mathrm{TiO}_{2}$ coated cotton fabrics with self-cleaning and UV blocking properties. Cellulose 264:2821-2832.

4. Akrman J, Přikryl, J. (2008). Application of benzotriazole reactive UV absorbers to cellulose and determining sun protection of treated fabric spectrophotometrically. J Appl Polym Sci 108:334-341.

5. Algaba I, Riva A(2002) Invitro measurements of theultraviolet protection factor of apparel textiles. Color Technol 118:52-58.

6. Ates ES, Unalan HE (2012) Zinc oxide nanowire enhanced multifunctional coatings for cotton fabrics. Thin Solid Films 52014:4658-4661.

7. Bojinov VB (2006) Synthesis of novel bifunctional hindered amine-UV absorber polymer stabilizers. Polym DegradStab 91:128-135.

8. Cheng $\mathrm{D}$ et al. (2018). Durable UV-protective cotton fabric by deposition of multilayer $\mathrm{TiO}_{2}$ nanoparticles films on the surface. Journal of Coatings Technology and Research 153:603-610.

9. Chen Z, Yin G. (2010). Suitability of a rare earth organic light conversion agent of Eu (III) complex to improve ultraviolet protection properties of cotton fabrics. Textile Research Journal 80: 1982-1989.

10. Choi SS, Song MJ (2008) Analysis of cyanoacrylate ultraviolet absorbers using liquid chromatography/atmospheric pressure chemical ionization mass spectrometry: influence of 
fragmentor voltage and solvent on ionization and fragmentation behaviors. Rapid Commun Mass Spectrom22:2580-2586.

11. Czajkowski W et al. (2006) Synthesis of reactive UV absorbers, derivatives of monochlorotriazine, for improvement in protecting properties of cellulose fabrics. Dyes Pigments 71:224-230.

12. Czajkowski W et al. (2012) Application of reactive UV-absorbers for increasing protective properties of cellulose fabrics during standard laundering process. Fibers and Polymers 137:948-953.

13. Dong L, Liu X, Xiong Z, Sheng D, Lin C, Zhou Y, Yang Y (2018a). Preparation and characterization of functional poly (vinylidene fluoride)(PVDF) membranes with ultraviolet-absorbing property. Applied Surface Science 444:497-504.

14. Dong L, Liu X, Xiong Z, Sheng D, Zhou Y, Lin C, Yang Y (2018b). Design of UV-absorbing PVDF membrane via surface-initiated AGET ATRP. Applied Surface Science 435:680-686.

15. Entezaria M, Shamelib A, Hekmatic M, Hekmatd S (2012) Synthesis and application of novel phenols as UV absorber in dyes and polymers. Trends Mod Chem 5:14-19.

16. Gorjanc M et al. (2014) UV protective properties of cotton fabric treated with plasma, UV absorber, and reactive dye. Polym.Fibers and polymers 1510:2095-2104.

17. Gouda M, Keshk S M A S (2010). Evaluation of multifunctional properties of cotton fabric based on metal/chitosan film. Carbohydrate Polymers 802:504-512.

18. Gupta V et al. (2021). Sol gel synthesis and application of silica and titania nano particles for the dyeing and uv protection of cotton fabric with madder. Journal of Natural Fibers 1-11.

19. Ibrahim HI et al. (2020) Synthesis, characterization and application of reactive UV absorbers for enhancing UV protective properties of cotton fabric. Egyptian Journal of Chemistry 632:525-536.

20. Ibrahim NA et al. (2013) Multifunctional finishing of cellulosic/polyester blended fabrics. Carbohydrate Polymers 972:783-793.

21. Mamnicka J, Czajkowski W (2012) New fiber-reactive UV-absorbers increasing protective properties of cellulose fibres. Cellulose 19:1781-1790.

22. Oda H (2011) Development of UV absorbers for sun protective fabrics. Text Res J 81:2139-2148.

23. Ren $\mathrm{G}$ et al. (2018). A simple way to an ultra-robust superhydrophobic fabric with mechanical stability, UV durability, and UV shielding property. Journal of colloid and interface science 522:57-62.

24. Rong $L$ et al. (2015) Multi-functional properties of cotton fabrics treated with uv absorber and $n-$ halamine. Fibers and Polymers169: 1876-1881.

25. Shen $Y$ et al. (2014). Improving anti-UV performances of cotton fabrics via graft modification using a reactive UV-absorber. Cellulose 215:3745-3754.

26. Shen Y, Zhu J, Chen H, Huang D (2016) Synthesis, characterization, and crystal structure of modified benzophenone uv-absorber containing reactive group. Research on Chemical Intermediates 424:2909-2918.

27. Stark NM, Matuana LM (2006) Influence of photostabilizers on wood flour-HDPE composites exposed to xenon-arc radiation with and without water spray. Polym Degrad Stab 91:3048-3056. 
28. Suhadolnik JC, DeBellis AD, Hendricks-Guy C, lyengar R,Wood MG (2002) Unexpected electronic effects on benzotriazole UV absorber photostability: mechanistic implicationsbeyondexcitedstateintramolecularprotontransfer. J Coat Technol 74:55-61.

29. Waiblinger $F$ et al. (1999). Ultraviolet absorbers and singlet oxygen. Journal of Photochemistry and Photobiology A: Chemistry 1261-3:43-49.

30. Wang $L$ et al. (2011). Superhydrophobic and ultraviolet-blocking cotton textiles. ACS applied materials and interfaces 34:1277-1281.

31. Zhao Y, DanY (2007) Synthesis and characterization of a polymerizable benzophenone derivative and its application in styrenic polymers as UV-stabilizer. European Polymer Journal 4310:4541-4551.

\section{Figures}

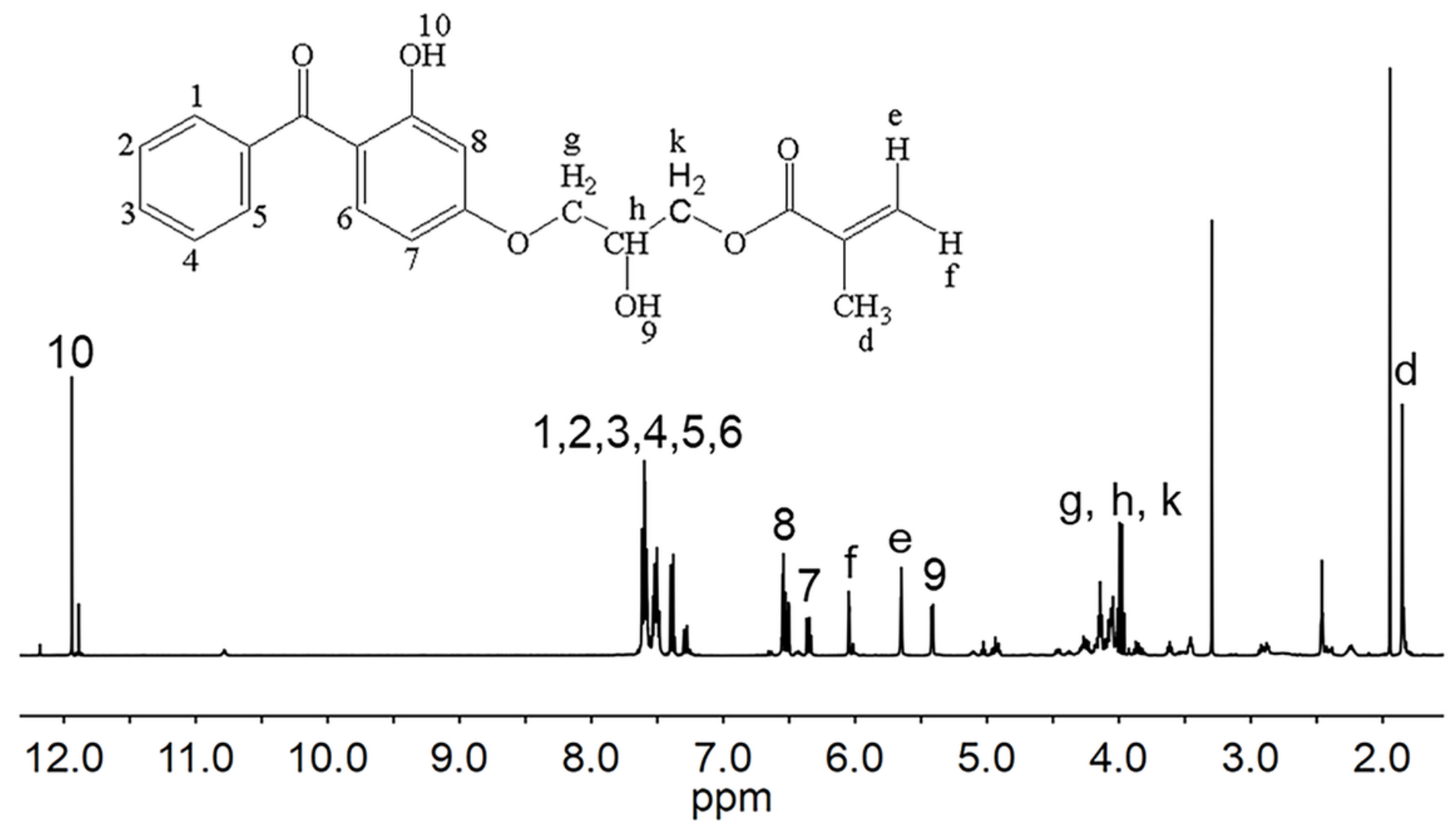

Figure 1

1H NMR spectrum of the BPMA in DMSO-d6 


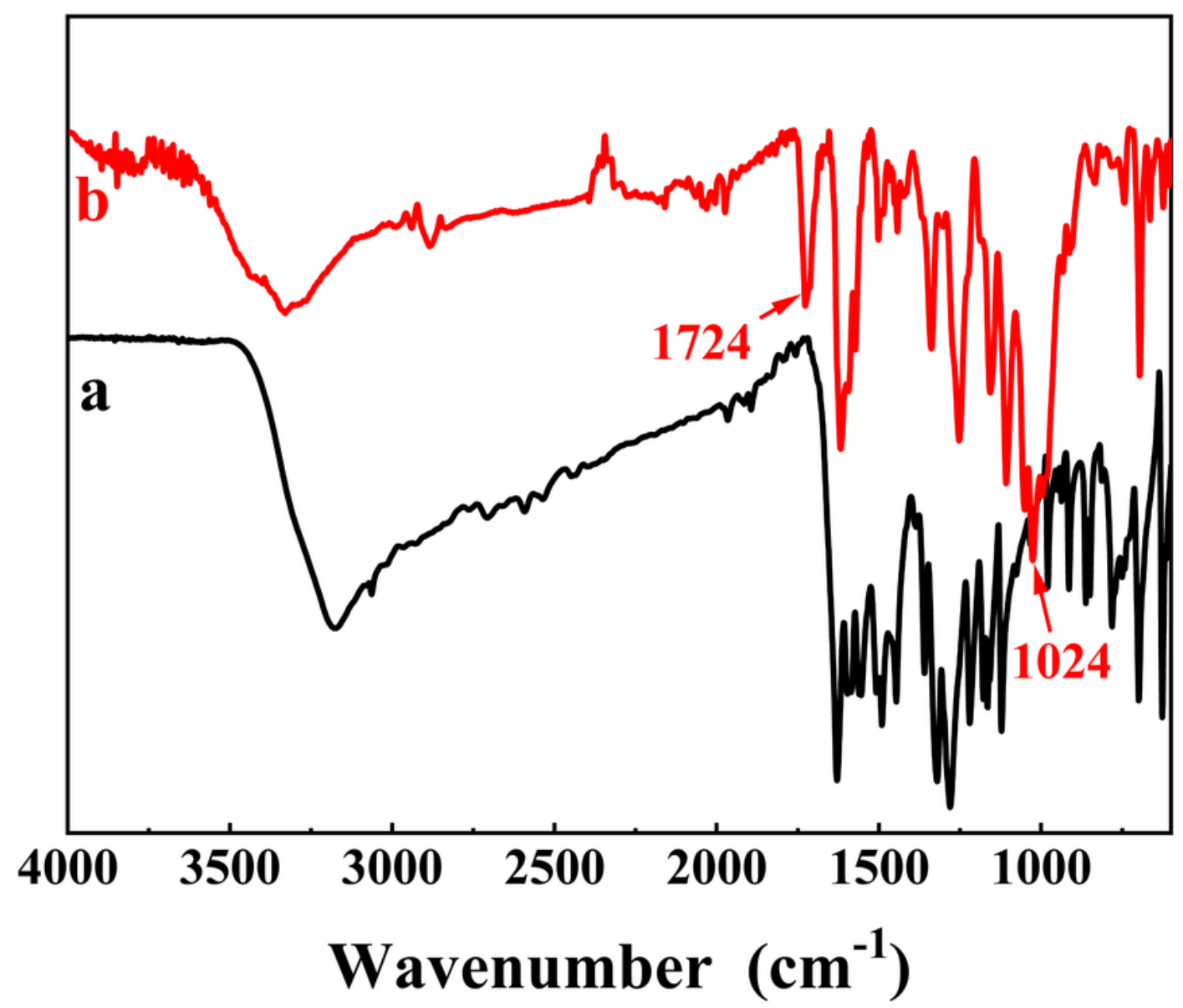

Figure 2

FT-IR spectra of UV-0 (a) and BPMA (b) (Note: all spectra have been corrected for atmospheric water and all were vertically offset for clarity). 


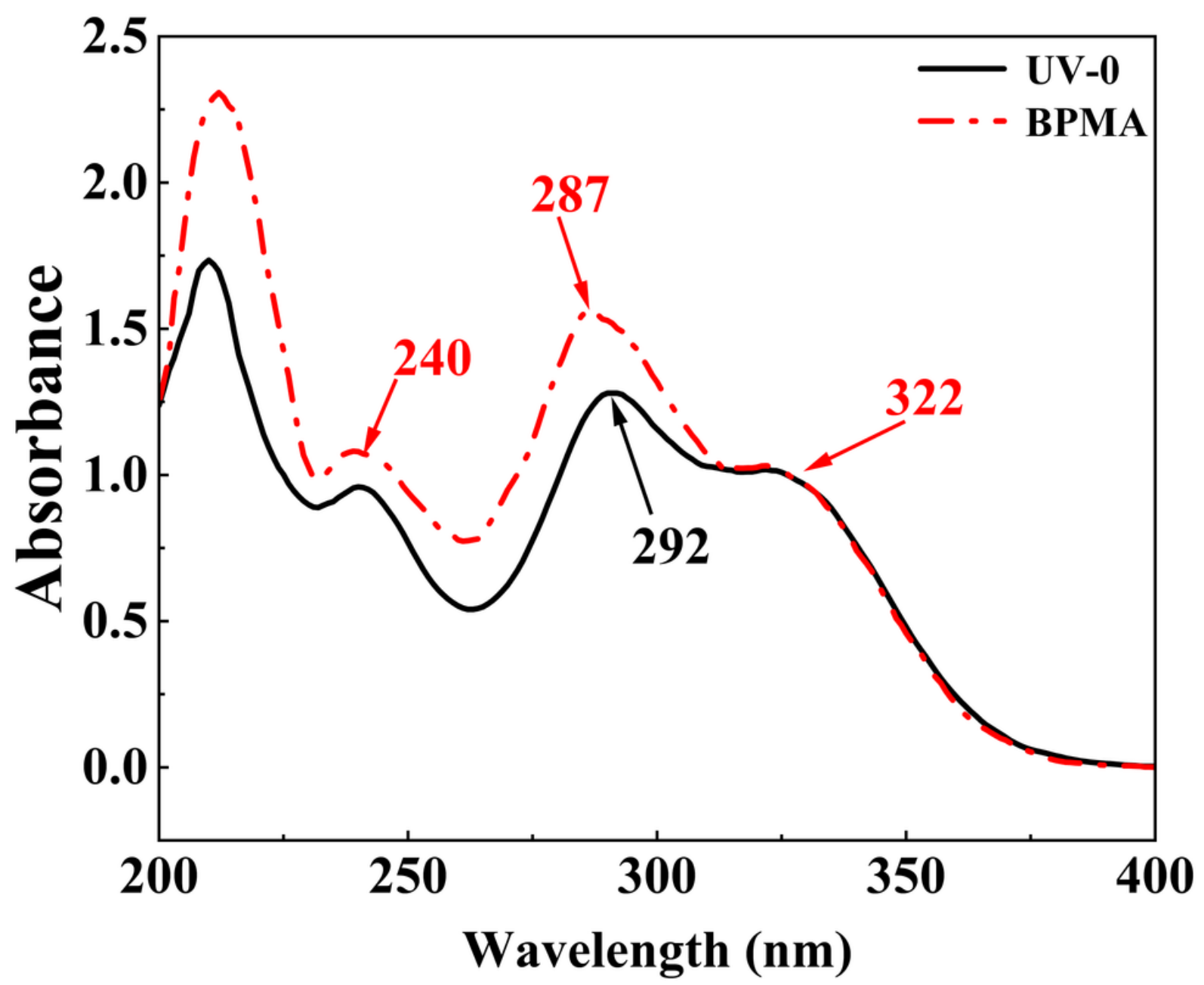

Figure 3

The UV-visible absorption spectra of BPMA and UV-0 (1×10-7 mol/ml in methanol). 


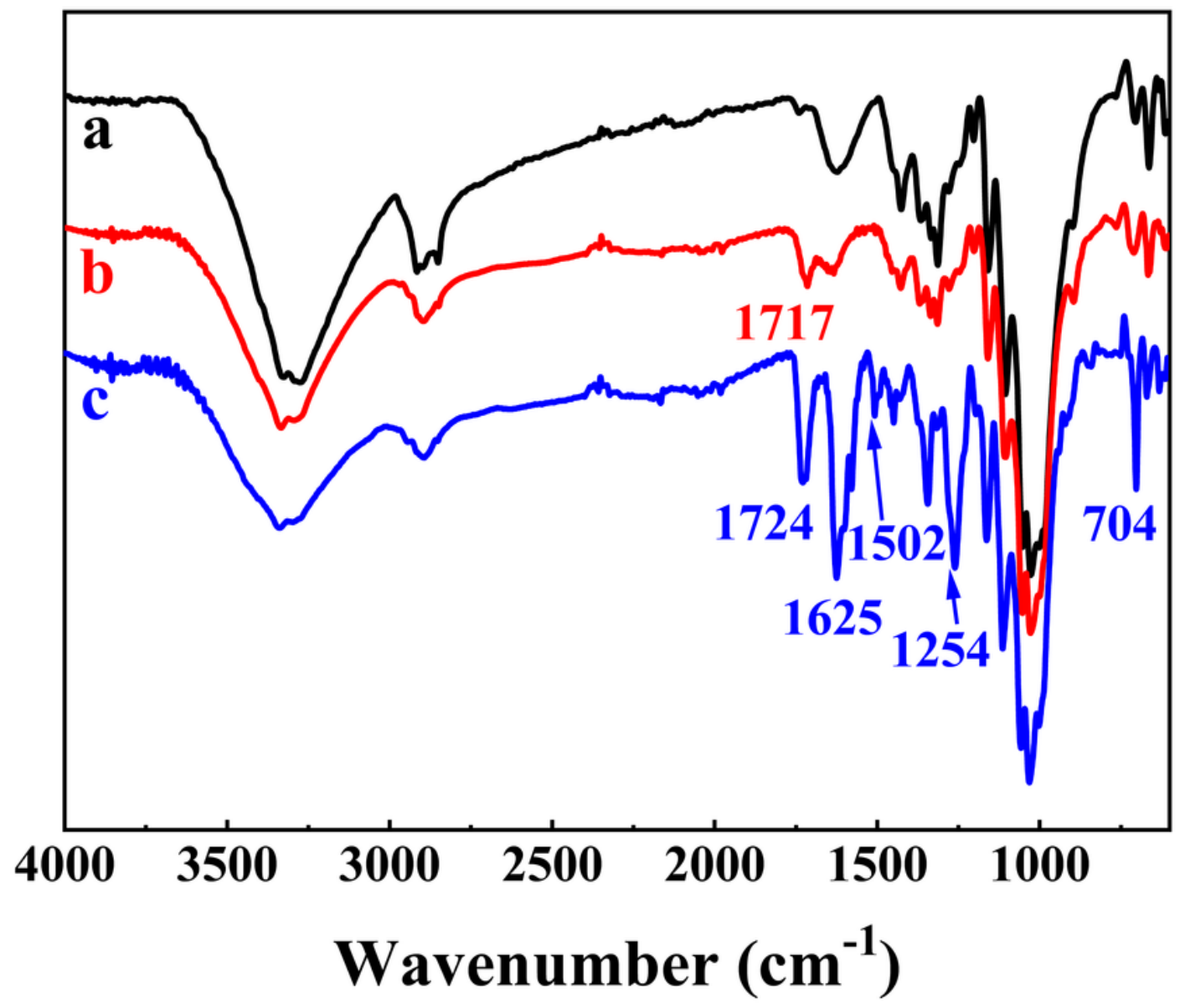

Figure 4

FT-IR spectra of original cotton fabric (a), CF-Br (b), and CF-g-PBPMA (c). (Note: all spectra have been corrected for atmospheric water and all were vertically offset for clarity.) 


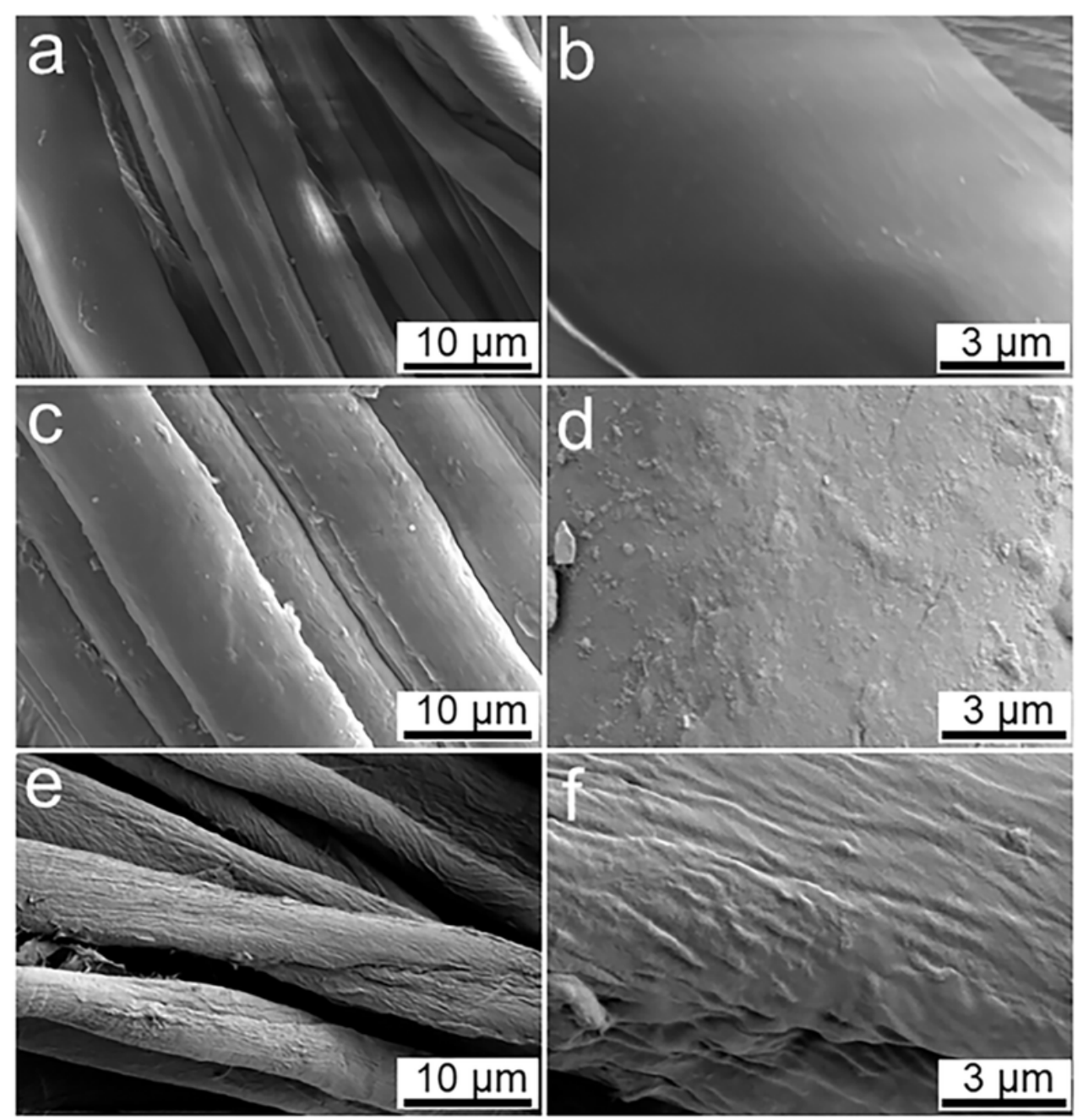

Figure 5

SEM images of original cotton fabric (a and b), CF-Br (c and d), and CF-g-PBPMA (e and f). 
(a)

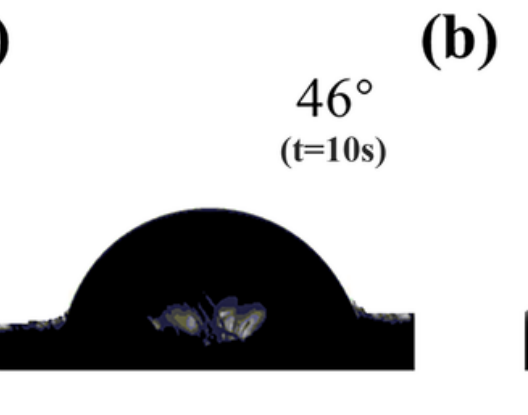

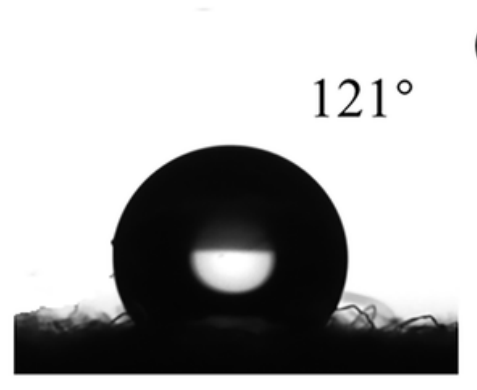

(c)

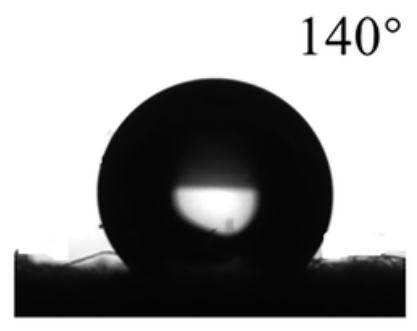

Figure 6

The WCAs of images original cotton fabric (a), WCA of CF-Br (b), and CF-g-PBPMA. 


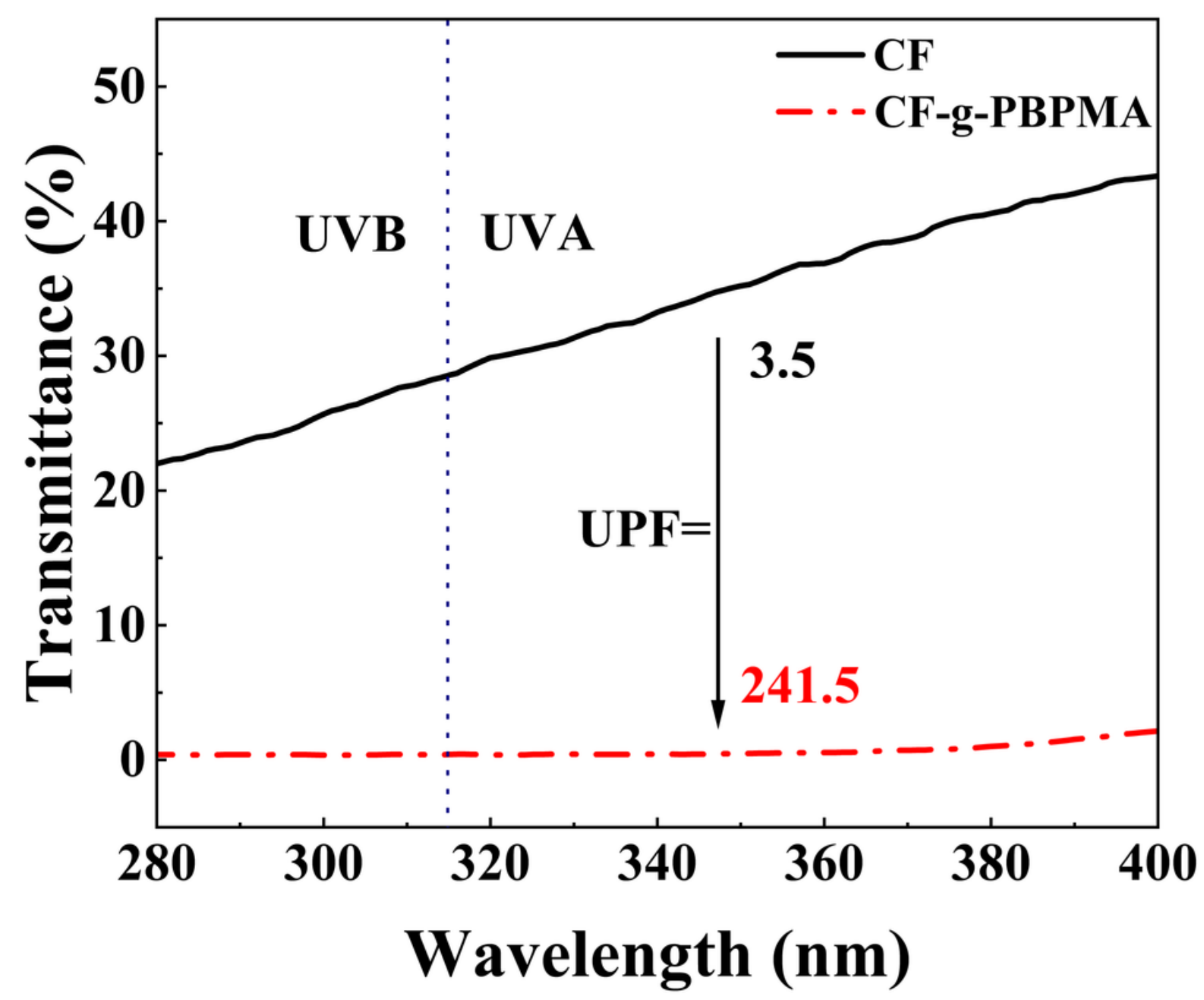

Figure 7

The UV-visible transmittance spectra of original cotton fabric (CF) and CF-g-PBPMA. 
(a)

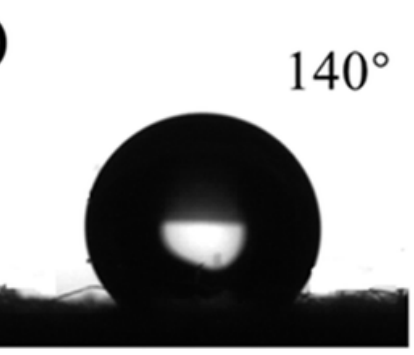

(b)

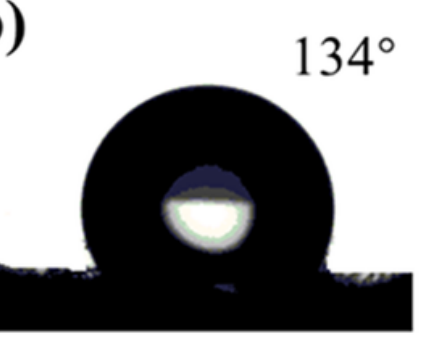

(c)

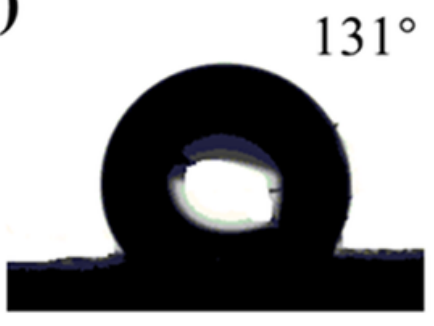

\section{Figure 8}

The WCAs of CF-g-PBPMA (a), CF-g-PBPMA after one cycle of washing (b) and CF-g-PBPMA after five cycles of washing (c). 


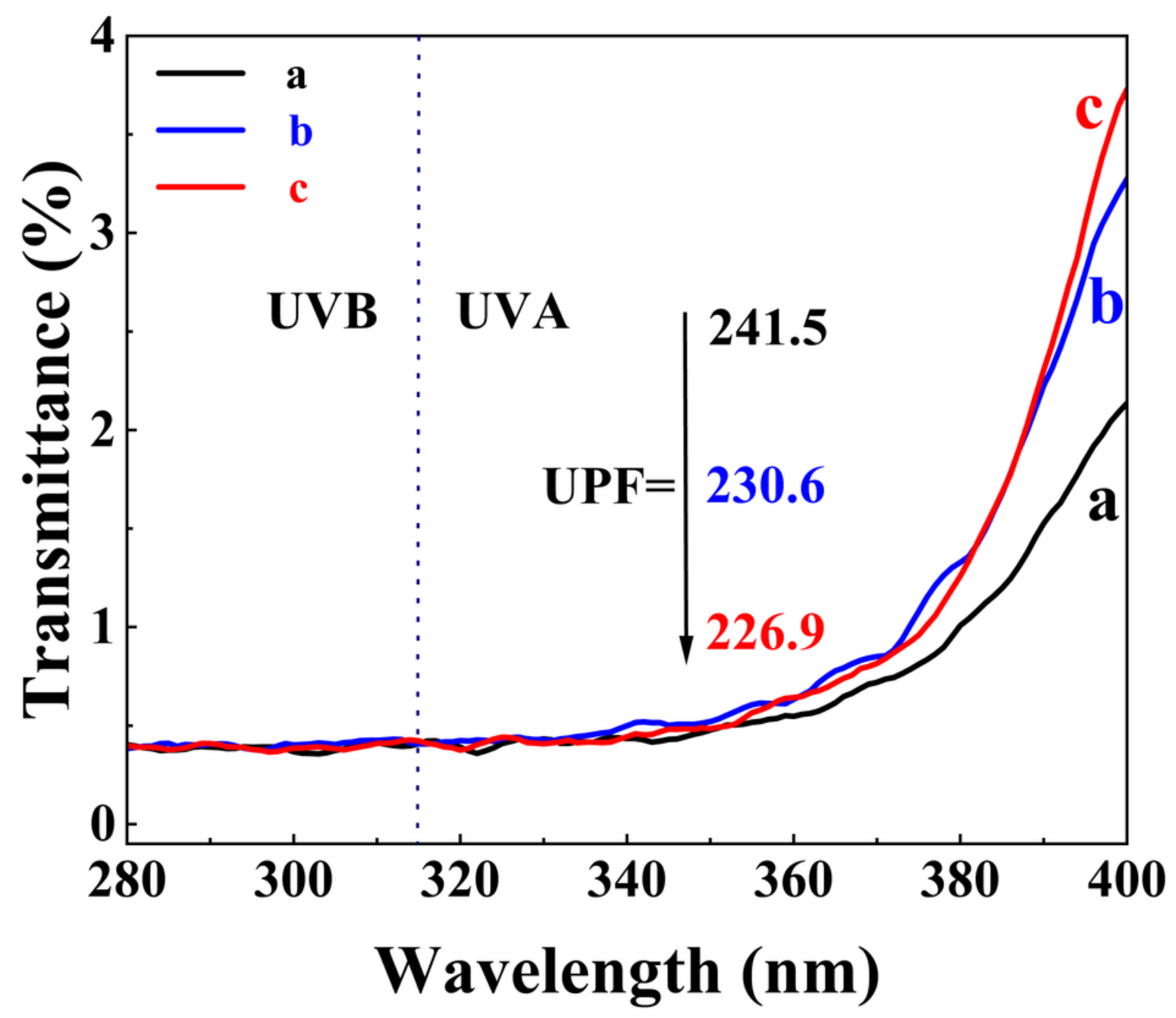

Figure 9

The UV-visible transmittance spectra of CF-g-PBPMA (a), CF-g-PBPMA after one cycle of washing (b) and CF-g-PBPMA after one/five cycles of washing (c). 


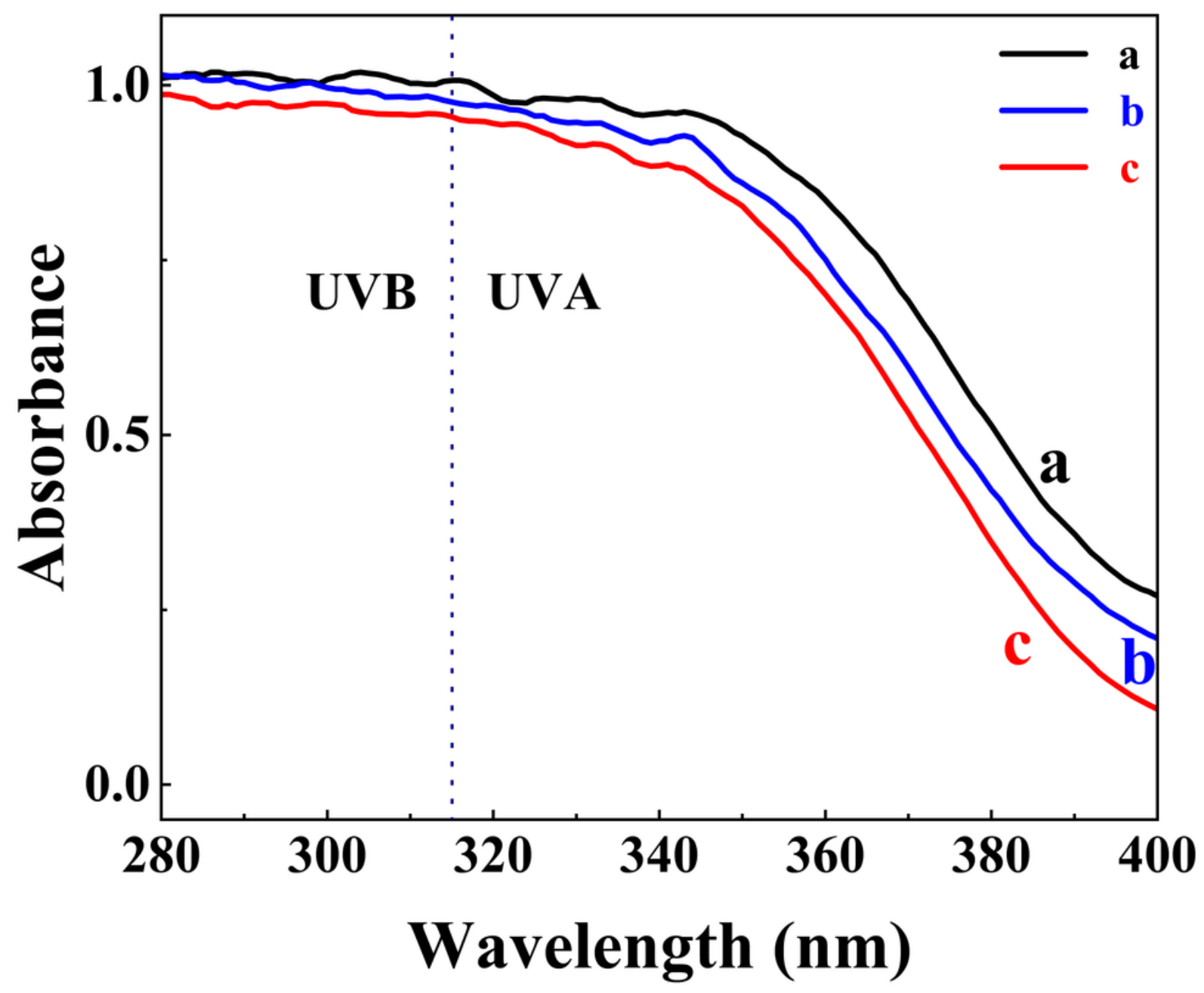

Figure 10

The UV-visible absorption spectra of CF-g-PBPMA (a), CF-g-PBPMA after one cycle of washing (b) and CF-g-PBPMA after five cycles of washing (c).

\section{Supplementary Files}

This is a list of supplementary files associated with this preprint. Click to download.

- Graphicabstract.png

- Scheme1.png

- Scheme2.png 